\title{
ELJUGADOR IMPLICADO EN EL CINE. ADAPTACIONES DE VIDEOJUEGOS A PELÍCULAS
}

\author{
Lluís ANYÓ \\ Universitat Ramon Llull
}

\section{RESUMEN}

La figura teórica del jugador implicado indica una característica fundamental de los videojuegos: su jugabilidad o relato dinámico. A partir de este concepto, se analiza distintos tipos de adaptación de los videojuegos al cine, de acuerdo con una definición amplia de la adaptación. Entendemos adaptación como un proceso de transformación de un lenguaje a otro, una traducción que puede ser directa o indirecta. Las adaptaciones, en el caso de los videojuegos al cine, pueden dividirse en tres tipos: a) adaptación del mundo de juego, que se convierte en universo diegético en el cine; b) adaptación de las reglas y estructura, que se convierte en estructura narrativa y c) adaptación de la participación y jugabilidad, que se convierte en estilo y puede tener un carácter alegórico. Esta perspectiva debería contribuir a un mejor conocimiento del cine contemporáneo.

Palabras clave: Adaptación, jugador implicado, mundo de juego, universo diegético, reglas, estructura narrativa, participación, jugabilidad, estilo, alegoría.

\section{ABSTRACT}

The theoretical figure of the implied player indicates a fundamental characteristic of videogames, their gameplay or dynamic narrative. From this concept, we analyze different types of adaptation of video games to the cinema, in a wide definition of adaptation. We understand adaptation as a process of transformation from one language to another, a translation that can be direct or indirect. Adaptations, from video games to cinema, can be divided into three types: adaptation of the game world, which becomes a diegetic universe in films, adaptation of rules and structure, which becomes narrative structure and adaptation of participation and gameplay, which can be a kind of style and an allegorical character. This perspective should contribute to a better knowledge of the contemporary films.

Keywords: Adaptation, implied player, game world, diegetic universe, rules, narrative structure, participation, gameplay, style, allegory. 


\section{CUESTIONES TEÓRICAS: EL CONCEPTO DE ADAPTACIÓN}

No intentaremos caracterizar aquí un fenómeno tan complejo y difícil de definir como es el del cine contemporáneo, cuyas transformaciones suponen al mismo tiempo la mayor diversificación histórica del lenguaje cinematográfico conocida hasta la fecha y también su crisis más duradera. Esta contradicción ya es palpable en la última década del siglo pasado, cuando la concentración empresarial en la industria de las Majors de Hollywood, que pasan a concebir sus productos como franquicias y blockbusters, impone un consumo global que no va a limitarse al producto estrictamente fílmico, sino que ya irreversiblemente debe incluir una expansión de productos conectados de las más diversas formas, a menudo a partir de un universo narrativo común, que se desarrolla en secuelas, precuelas, spin-offs, nuevas versiones de todo tipo, reboots, productos transmedia y crossmedia, atracciones de parque temático, objetos resultado del licensing y multitud de otras mutaciones más o menos narrativas y sobretodo comercialmente viables.

Como resultado, el cine contemporáneo -también el de las producciones más independientes, que necesitadas de una fuerte comunidad de apoyo, muchas veces económico, tirarán de su expansión en redes sociales- no puede analizarse sólo en la película aislada, sino en relación con los productos que componen su universo narrativo y comercial.

Es en este sentido que se ha hablado del Nuevo Hollywood ((Tasker, 1998); (Riambau, 2011)), del cine posmoderno (Deleyto, 2003), de la pantalla global (Lipovetsky \& Serroy, 2009) o directamente de las adaptaciones en su más amplio alcance (De-Felipe \& Gómez, 2008).

Efectivamente, para Lipovestsky y Serroy, la pantalla global

remite al nuevo dominio planetario de la pantallasfera, al estado-pantalla generalizado que se ha vuelto posible gracias a las nuevas tecnologías de la información y la comunicación. Son los tiempos del mundo pantalla, de la todopantalla, contemporánea de la red de redes, pero también de las pantallas de vigilancia, de las pantallas informativas, de las pantallas lúdicas, de las pantallas de ambientación. El arte (arte digital), la música (el videoclip), el juego (el videojuego), la publicidad, la conversación, la fotografía, el saber: nada escapa ya a las mallas digitalizadas de esta pantallocracia. La vida entera, todas nuestras relaciones con el mundo y con los demás pasan de manera creciente por multitud de interfaces por las que las pantallas convergen, se comunican y se conectan entre sí (Lipovetsky \& Serroy, 2009: 22-3).

De modo que

en la cultura hipermoderna hay algo que sólo se puede llamar espíritu cine y que atraviesa, riega y nutre las demás pantallas: el cine se ha convertido en un círculo cuyo centro está en todas partes y circunferencia en ninguna. Cuanto más compiten con él o lo sustituyen la Red, la televisión, los videojuegos y los espectáculos deportivos, más fagocita su estética esencial áreas enteras de la cultura de la pantalla. Por los espectáculos, por los deportes, por la televisión y un poco por todas partes cabalga hoy el espíritu del cine, el culto a lo visual espectacularizado 
y a los personajes famosos. Infinitamente más poderoso y global que su universo nativo y específico, el cine parece hoy la matriz de lo que se expresa de él (Lipovetsky \& Serroy, 2009: 25).

Hagamos notar que los videojuegos aparecen una y otra vez en el conjunto de esta pantalla global de la comunicación contemporánea.

No es la pantalla global la única denominación del estado actual de lo audiovisual, aunque quizá sea la que ha tenido más éxito académico. Con otro término más clásico, pero al menos igualmente sugerente, F. de Felipe e I. Gómez inscriben el momento contemporáneo del cine en el campo de las adaptaciones, auténtico concepto central para entender las transformaciones del medio:

Al guionista, como el curioso impenitente que debería ser, se le acabará exigiendo que desarrolle por su cuenta y riesgo una cada vez más panorámica visión del mercado, lo que le obligará a estar siempre al día en lo que respecta tanto a las dinámicas y funcionamientos internos de campos tan diversificados como puedan serlo el cómic, la novela, la ficción televisiva, los videojuegos o el propio cine, como a la propia actualidad (fuente constante de inspiración). Y todo esto sin olvidar que, hoy en día un buen guionista es aquel que entiende que una película, propia o de encargo, no es sino el primer producto de una larga cadena de historias subsidiarias que tenderán a multiplicarse exponencialmente hasta el infinito de cara a poder ser distribuidas (y amortizadas) en todos y cada uno de los soportes de los que dispon(dr)emos. Y es que lejos de restarle importancia a la obra cinematográfica, lo que la actual proliferación de pantallas, soportes, formatos y canales de distribución de imágenes ha terminado generando es el fácil acceso de creadores y espectadores a unas hasta ahora inéditas armas de (di)fusión masiva (De-Felipe \& Gómez, 2008: 24).

De acuerdo con lo dicho hasta ahora, consideraremos adaptación el proceso de transformación de un determinado texto, o conjunto de textos de un medio concreto, soporte de un determinado contenido ficticio, narrativo o no, de una estructura y de una forma, en un texto similar en algún sentido, pero cuyo contenido, estructura y forma se expresan en el soporte de un medio distinto.

Aunque centrados en el análisis de los textos o productos, queremos dar cuenta de cambios que afectan al modo en que el consumidor accede a los contenidos de ocio, más allá de su pertenencia cultural, su soporte o formato e incluso su medio original, sea la literatura, el cómic, la televisión, el propio cine o por supuesto los videojuegos.

Felizmente instalado en la encrucijada intermediática del nuevo milenio, el espectador contemporáneo sabe que, hoy por hoy, las historias más emocionantes pueden manifestarse en casi cualquier sitio. Acostumbrado a saltar de un medio a otro con la libertad del que se sabe no sujeto a compromiso ontológico alguno, el espectador contemporáneo sigue el rastro de las ficciones de calidad hasta dar con ellas. Y lo mismo le da que éstas se encuentren en una película clásica o en una de culto, en un best-seller o en una antología de cuentos, en una obra de teatro o en un cómic de autor, en una serie norteamericana o en un videojuego japonés, en 
las noticias de la tele o en un documental histórico, en el videoclub de la esquina o colgadas en YouTube (De-Felipe \& Gómez, 2008: 30).

En definitiva, vamos a manejar en este artículo un concepto amplio de adaptación, inscrito en las formas contemporáneas del cine, pero por cuestiones de espacio, limitado no sólo a dos medios muy concretos, el cine y los videojuegos, sino también a una única dirección entre ellos, es decir, las adaptaciones que el cine ha llevado a cabo desde el ámbito de los videojuegos.

\section{METODOLOGÍA: EL JUGADOR IMPLICADO Y EL ESPECTADOR CINEMATOGRÁFICO}

Las relaciones y trasvases entre cine y videojuegos son sin duda innumerables y complejas. Tanto en una dirección como en otra, ambos medios comparten en la mayor parte de sus creaciones puntos en común estéticos y narrativos, de formas y de contenidos, de arte y de industria, y pueden considerarse ambos medios fundamentales para entender la comunicación audiovisual. Hemos defendido en otro sitio (Anyó, 2014) que los videojuegos deben incorporarse al campo disciplinar de la comunicación audiovisual, en tanto que se trata de representaciones audiovisuales, de acuerdo con la muy fructífera definición de representación de Manovich (2005: 165-6).

Desde luego, también las diferencias entre cine y videojuegos son más que notables y en ningún caso pretendemos que los videojuegos deban someterse al cine, en cuanto a su análisis o proceso creativo. Al contrario, queremos demostrar aquí que las distintas formas de adaptación entre uno y otro medio indican una estrecha relación expresiva al mismo tiempo que características distintivas. Para ello analizaremos distintas adaptaciones de los videojuegos al cine, para explorar el modo en que los primeros han influido, a estas alturas de la partida ya de forma irreversible, en los modos formales y en los contenidos de este último medio.

Los videojuegos son distintos del cine, sobre todo por dos características fundamentales que derivan de que se trata de artefactos regulados por reglas: la jugabilidad y la tematización. Aarseth propone una excelente guía metodológica para el análisis de los videojuegos. Divide todo juego en tres aspectos: sus reglas, su mundo de juego y su jugabilidad. Juul por su parte denomina tematizable [themeable] a la cualidad de los videojuegos que permite asignar diferentes mundos de ficción al mismo conjunto de reglas $(2005,199)$. Efectivamente, y de acuerdo con ambos autores, los videojuegos son artefactos culturales que funcionan en base a reglas que permiten la manipulación lúdica de entornos virtuales, entendidos éstos como entornos distintos del entorno de la vida. El concepto de manipulación lúdica remite a la jugabilidad, característica fundamental de todo videojuego, puesto que da cuenta de su carácter participativo.

Entonces, es posible modificar el mundo de juego sin que podamos hablar propiamente de un juego distinto, como es el caso de los juegos de ajedrez -mismas reglas- con distintos diseños de piezas -mundo de juego-. En palabras de Aarseth, «the dimension of Lara Croft's body, already analyzed to death by film theorists, are irrelevant to me as a player, because a different-looking body would not make me play differently» (2004: 48). Aunque estamos de acuerdo en la importancia del concepto de tematización, no lo estamos con la generalización de considerar irrelevante el mundo de juego. Al contrario, en los videojuegos narrativos como Tomb Raider (1996-2015), aunque el mundo de ficción no forma parte de las 
reglas del juego, afecta a distintos aspectos diegéticos - un determinado personaje, viviendo un determinado tipo de aventura, etc.- y también no diegéticos, en particular, el efecto que aquéllos puedan tener en el espectador, en forma de distintas emociones: si siento miedo al jugar a Until Dawn (2015), no es por sus reglas o estructura, sino por el hecho de enfrentarme como jugador, a través de un personaje, a otros personajes -que remiten al cine de terrorque por su aspecto gráfico, comportamiento, etc., me provocan escalofríos.

En cuanto a la jugabilidad, la consideramos un aspecto fundamental de todo videojuego, en tanto que es en las partidas donde podemos considerar la concreción del relato de un videojuego, distinto cada vez, a diferencia de narrativas no participativas como el cine. La jugabilidad produce un relato que podemos llamar dinámico. Aarseth define la jugabilidad como las acciones, estrategias y motivaciones del jugador durante su interacción con el juego. Es un elemento extremadamente volátil en el análisis de los videojuegos, pero también central para la comprensión de sus mecanismos. Como hemos defendido en otro sitio, el jugador implicado crea el relato en cada una de sus partidas, que remiten a esquemas de acción, estrategias y posibilidades, en directa relación con las mecánicas del juego y que constituyen auténticas activaciones de las reglas. El jugador implicado juega partidas implicadas, que son lo más próximo en videojuegos al relato en literatura o cine (Anyó, 2016: 50).

El jugador implicado es entonces distinto del espectador cinematográfico, cuya pasividad suele contraponerse y quizá exagerarse en relación a la acción del jugador en el juego. Desde luego, podemos decir que el espectador cinematográfico interviene en algún sentido en el film, por ejemplo, como haría notar Hitchcock a Truffaut, en las películas de «suspense»: «En el estreno de Rear Window, yo estaba sentado al lado de la mujer de Joseph Cotten y, en el momento en el que Grace Kelly registra la habitación del asesino y éste aparece en el pasillo, ella estaba tan inquieta que se volvió a su marido y le dijo: "Haz algo, haz algo"» (Truffaut, 1984: 68).

También la inmersión y la identificación, mecanismos compartidos por el cine y los videojuegos, se encuentran en relación con esta actitud atenta y hasta cierto punto activa del espectador en la ficción cinematográfica. Ambos mecanismos implican una relación del espectador con el texto, en la medida que la inmersión es «la experiencia de trasladarse a un lugar ficticio muy elaborado» (Murray, 1999: 111) y la identificación, por ejemplo, la identificación secuntaria de Metz (2001) o la participación afectiva de Morin, es la

necesidad de escaparse, es decir, de perderse en otra parte, de olvidar su límite, de participar mejor en el mundo. Es decir, a fin de cuentas, de escaparse para volverse a encontrar, Necesidad de volverse a encontrar, de ser más uno mismo, de elevarse a la imagen de ese doble que lo imaginario proyecta en mil vidas asombrosas. Es decir, necesidad de volverse a encontrar para escaparse, volverse a encontrar en otra parte distinta a nosotros mismos, escaparse al interior de nosotros mismos. La «especificidad» del cine, si así puede decirse, es ofrecer la gama potencialmente infinita de esas huidas y reencuentros; el mundo al alcance de la mano, todas la fusiones cósmicas y también, la exaltación en el espectador de su propio doble encarnado en los héroes de amor y de la aventura (Morin, 2011: 104).

Sin embargo, y aunque estos mecanismos se mantienen también en los videojuegos, la diferencia fundamental que indicamos con el término de participación no pertenece al 
cine, puesto que en el cine el relato está dado de antemano, independientemente de la experiencia de inmersión e identificación del espectador, mientras que en los videojuegos el relato no solo puede ser modificado por el jugador, sino que es necesario que sea jugado para convertirse en relato -relato dinámico- y de tal modo que cada partida, del mismo o de distintos jugadores, será inevitablemente distinta, pero sin que podamos reducir el videojuego a un texto prefijado, puesto que éste no existe en su carácter perdurable de reglas y gráficos, sino que existe una vez y cada vez que es jugado por el jugador, quien, como se ve, tiene un papel creativo fundamental, el de generador del relato del videojuego. Este jugador implicado, que se encuentra por tanto presente en el juego una vez jugado, es una figura teórica ineludible del análisis de los videojuegos. Como hemos dicho en otro sitio, «el jugador implicado activa las reglas y las convierte en juego a través de la jugabilidad [...]. El análisis narrativo de los videojuegos debe poner en un lugar central la relación entre mecánicas y jugabilidad, en clara diferencia con el cine. Aun cuando el jugador no es el creador o autor del juego, su acto de jugar, su experiencia y sus partidas constituyen el juego en sí mismo» (Anyó, 2016: 46-7).

En estas condiciones abordaremos las relaciones entre videojuegos y cine en una perspectiva amplia, considerando distintos tipos de adaptaciones que por cuestiones de espacio limitaremos a la dirección menos explorada en la literatura especializada: de los videojuegos al cine. Clasificaremos, por tanto, las influencias de los videojuegos en las películas en tanto se trata de adaptaciones del mundo de juego o mundo de ficción en tanto que se tratará logicamente de videojuegos narrativos, adaptaciones de las reglas y las estructuras o adaptaciones de la jugabilidad y la participación.

\section{RESULTADOS: TRES DISTINTOS TIPOS DE ADAPTACIÓN}

\subsection{Adaptaciones del mundo de juego}

El tipo de adaptación más evidente entre los textos de dos medios distintos, ambos narrativos, es el de la adaptación de la historia y contenido narrativo. Cuando pensamos en la adaptación de una novela al cine, en primer lugar pensaremos en la traslación de unos determinados espacio y tiempo, unos personajes concretos y unas acciones que éstos llevan a cabo. La fidelidad, concepto a menudo ligado al tema de las adaptaciones, se juzga sin duda sobre la similitud o no entre estos distintos aspectos del contenido argumental entre una y otra versión. También cuando pensamos en adaptaciones entre los videojuegos y el cine, en primer lugar pensamos en la traducción de un medio a otro, y en las dificultades que entrañan las diferencias de lenguaje que hemos visto en el apartado anterior, de unos determinados acontecimientos o narración de hechos. El mundo de juego, por tanto, en la medida en que se considera "adaptable», es el de juegos narrativos, es decir, un mundo de ficción equivalente al mundo diegético de la ficción cinematográfica. Para una discusión completa del mundo de ficción en los videojuegos puede verse A.J. Planells (2015).

Desde nuestro punto de vista, la adaptación del mundo de juego de un videojuego se traduce básicamente en la diégesis o historia narrada en una película y en un sentido más amplio en su universo diegético. Es relativamente fácil, en este sentido, establecer un corpus de películas que, de forma más o menos afortunada, proponen una determinada lectura y traducción de un mundo a otro, con una mayor o menor fidelidad con respecto al original, en el caso de los videojuegos a menudo adaptación de distintas entregas de un 
mismo título genérico. Este es el caso de Super Mario Bros (1993), Double Dragon (1993), Street Fighter: la última batalla (1994), Mortal Kombat (1995), Resident Evil (2002), Hitman (2007), Max Payne (2008), Prince of Persia: las arenas del tiempo (2010), The King of Figther (2010), Tekken (2010), Need for Speed (2014), Final Fantasy: la fuerza interior (2001), Postal (2007), Alone in the dark (2005), Silent Hill (2006) y Lara Croft: Tomb Raider (2001), entre otras, películas todas ellas que corresponden a títulos concretos o los títulos de una serie de una misma saga. Efectivamente, esta es la perspectiva por ejemplo empleada por F. de Felipe e I. Gómez en el capítulo dedicado a los videojuegos, que analiza la adaptación cinematográfica de la serie de videojuegos del mismo título en la película Silent Hill (DeFelipe \& Gómez, 2008: cap. 4).

Sin embargo, la adaptación en términos de mundo de juego puede verse también en relación no ya con una historia o diégesis más o menos concreta, sino en relación con un cierto universo diegético que inspira la adaptación o que es compartido por ambos productos. Sería el caso de Assassin's Creed (2007) y El reino de los cielos (2005) en relación con la Tierra Santa de las Cruzadas y la familiaridad de ciertos ambientes y personajes (Villalobos, 2014: 38) o L.A. Norie (2011) y las novelas de James Ellroy y clásicos y modernos del cine negro como El halcón maltés (1941) o L.A. Confidencial (1997) (Villalobos, 2014: 45).

Centrémonos con un cierto detalle en la adaptación de la serie Tomb Raider, analizada de forma pormenorizada por M-P. Martínez-Fabre (2017) en su artículo sobre adaptaciones. Para Martínez-Fabre, el éxito comercial de la adaptación, después de una serie de lamentables fracasos de intentos anteriores por llevar a la pantalla cinematográfica distintos videojuegos, puede explicarse en parte por inscribirse en una serie de productos-videojuegos y películasque conforman una red de autoreferencialidades basadas en el mundo de ficción, es decir, personajes aventureros, escenarios salvajes, búsqueda de tesoros, etc., muy propios del cine clásico y postmoderno de aventuras y también de la serie Tomb Raider:

Otro aspecto que nos aproxima al éxito de Lara Croft: Tomb Raider es que la película, aunque algo prosaica, es una recreación muy próxima al juego que explota acertadamente la vertiente cinematográfica de éste mismo. Su cóctel de acción, mitología y sucesos paranormales (prácticamente calcado de las aventuras del citado Indiana Jones) se adapta sin problemas a un guion del cine espectáculo. Además, en su trasvase cinematográfico respeta el texto original y celebra los clichés estéticos que le dieron fama, en mayor medida que anteriores producciones de alto presupuesto como Super Mario Bros, o Street Fighter. De partida, este aspecto fílmico del juego es una gran ventaja: en comparación al resto de las anteriores adaptaciones, el film parte de un juego que ya poseía un notable espíritu cinematográfico inspirado en los films de aventuras del cine espectáculo tales como los del Indiana Jones o los del agente James Bond (Martínez-Fabre, 2017).

Martínez-Fabre sitúa acertadamente esta película como giro de tendencias hacía adaptaciones de alto presupuesto rentables para la industria:

La clave principal del film es la articulación del cine de acción/aventuras junto a la confluencia "personificada» de dos explosivas figuras: la del personaje de videojuego Lara Croft, y la de la actriz destinada a encarnarla, Angelina Jolie. Lara Croft es un personaje diversamente atractivo tanto dentro como fuera del 
espacio del videojuego. Su reinterpretación visceral de la figura del aventurero (en especial del personaje de Indiana Jones creado por George Lucas y Steven Spielberg) en una forma más violenta y más sexual, era ya un reclamo de éxito en la saga de juegos y tenía todas las cualidades para triunfar en un film de sensaciones del tipo cine espectáculo. Más, con el añadido interpretativo de una actriz tan voluptuosa como Angelina Jolie; anzuelo morboso para los fans y los no entendidos del videojuego. Parte del atractivo de esta suma Croft-Jolie reside, sin embargo, en la masculinización temperamental a la que se somete su propio estereotipo femenino. Lara pasa de ampararse vigorosamente en símbolos fálicos [...] a mostrarse sensualmente desnuda mientras se ducha o mientras porta ceñidos atuendos que acentúan sus «hipersexualizadas» curvas. Además, corretea por el film, rodeada por un amplio grupo de actores «masculinos» [...], que apenas deja espacio a otras figuras femeninas (Martínez-Fabre, 2017).

En efecto. Fijémonos, para los propósitos de nuestro argumento, en que la adaptación Lara Croft: Tomb Raider, ya claramente indicada en el título de la película, utiliza un reclamo diegético de la serie de videojuegos: el personaje virtual de Lara Croft, perfectamente personalizado en el personaje extradiegético de Angelina Jolie, para, en un perfecto engranaje intertextual e intermedial, adaptar los siguientes videojuegos de la saga a la figura mediática de la actriz.

La presencia absoluta de Angelina Jolie, como se ha comentado, funciona tanto para ensalzar la figura icónica de Lara Croft, como para explotar el reclamo del film. Visto el éxito de esta elección, Jolie repetirá posteriormente en la secuela Tomb Raider: La Cuna de la Vida (Jan de Vont, 2003), y marcará un precedente en próximas adaptaciones de videojuegos (tal y como hacen otras estrellas dentro del cine espectáculo). Si ella ocupó el metraje de Lara Croft, Mila Jovovich lo hará en la saga de films Resident Evil, Jake Gyllenhaal en Prince of Persia: Las Arenas del Tiempo, o Mark Walhberg en Max Payne. Todos ellos son «estrellas» mediáticas atractivas para el público y, a su vez, capaces de asumir y permutar el rol icónico de un personaje de videojuego. Cabe señalar que las figuras de Jolie, Jovovich, Gyllenhaal, etc. han aportado y competido en la pantalla de cine tanto por el personaje que interpretan, como lo ha hecho su propia versión digital en el juego. El personaje digital de Lara Croft, fue remodelado en la siguiente entrega de la saga de juegos para asemejarse a Angelina Jolie, la figura de Mila Jovovich ha dado más fama (y más rostro) a la saga Resident Evil que muchos otros de los protagonistas virtuales de los juegos, etc. (Martínez-Fabre, 2017).

\subsection{Adaptaciones de las reglas y estructuras}

La adaptación en términos de reglas y estructuras buscará relacionar las reglas del videojuego, que a través de determinadas mecánicas proporciona la estructura del juego, con la estructura narrativa de la película. Como hemos dicho, un juego debe ser jugado, de manera que, a través de sus mecánicas, un juego determina una serie de posibilidades de progreso en su desarrollo, una estructura, y hace inviables otras, pero solo se concreta una trama fija cuando la partida es jugada. Esta concepción del jugador, que llamamos jugador implicado, 
confiere a éste una cierta responsabilidad narrativa, en tanto que genera el relato con su juego. También en el cine el relato depende de una instancia narrativa, real o ficticia, y de una serie de reglas narrativas más o menos explícitas -un equilibrio entre las expectativas del espectador y la originalidad necesaria en la narración, una relativa integración temática y estructural al género, etc.-, aunque, por supuesto, no es el espectador quien finalmente da forma al relato cinematográfico, sino que éste está dado de antemano a su visionado. En los videojuegos, la trama es un determinado relato en forma de partida que depende al mismo tiempo de las reglas y de las acciones del jugador. Por lo tanto, el tipo de adaptación que aquí se propone asimila el relato cinematográfico a las reglas del videojuego.

Nos encontramos entonces con dos soluciones de adaptación, por lo que respecta a la adaptación fílmica de un videojuego:

La primera de ellas nos obligaría a la construcción de una historia tradicional, un relato cinematográfico clásico que implique una cierta idea de clausura, continuidad y coherencia, eliminando del guion todo aquello que tenga que ver con la propia naturaleza interactiva del juego original y lo aleje de su canónica progresión dramática (repeticiones, bucles narrativos, desarrollos potenciales, finales alternativos, reinicios, tiempos muertos, elevación del grado de dificultad, etc.). [...] La segunda solución haría referencia a la construcción de un entorno (falsamente) interactivo que intentaría, en la medida de lo posible, traducir a términos estrictamente cinematográficos la experiencia del jugador [...]. Proceso de traducción para el que sería necesaria tanto la renuncia expresa a cualquier intento de articulación del relato resultante a la manera clásica [...], como la decidida apuesta por la (re)construcción de un entorno visual interactivo mucho más ambiguo y posibilista (De-Felipe \& Gómez, 2008: 211).

Aunque planteadas así parecen soluciones radicalmente distintas, haremos un par de consideraciones. En primer lugar, muchos videojuegos, sobre todo los del género de la aventura gráfica, tienen estructuras narrativas notablemente lineales, de modo que la interactividad, que la hay, no afecta tanto a la estructura como a las dificultades para avanzar por ella. Se trata de lo que en otro lugar hemos llamado narración progresiva (Anyó, 2016: 133) y se ejemplifica bien en el juego The Last of Us (2013), perfectamente analizable como guion cinematográfico según el esquema del paradigma esquemático y su línea argumental dividida en dos nudos de trama y tres actos (Colom \& Anyó, 2015).

Por supuesto, y a este grupo de adaptaciones nos referimos aquí, determinadas películas parecen tomar una forma intradiegética de la participación -como no puede ser de otro modo-, y proponen entonces distintos tipos de juegos narrativos, donde de la mano a menudo de un narrador cinemático no fiable, el espectador entra en los mundos de ficción que pueden ser, en ese mismo universo diegético, mundos alternativos, universos paralelos, alucinaciones, bifurcaciones que posibilitan otros acontecimientos, versiones distintas de la misma historia, bucles o repeticiones, etc. Se trataría por ejemplo de El club de la lucha (1999), Memento (2000), Femme Fatale (2002), Identidad (2003), Swimming Pool (2003), Mulholland Dr. (2002), Carretera perdida (1997), Funny Games (1997), Dos vidas en un instante (1998) o Donnie Darko (2001).

Un conjunto más restringido de este tipo de juegos narrativos haría referencia, de forma más directa, a las reglas propias de los videojuegos y su conversión en relato fílmico. Se trata 
entonces de películas que proponen caminos que se bifurcan -Run, Lola, Run (1998), The rules of attraction (2002)- una progresión en niveles de dificultad -eXistenZ (1999), Sucker Punch (2011)-, o la figura tan característica de los videojuegos del bucle que se repite hasta superar el nivel -Source Code (2011), Al filo del mañana (2014)-.

La adaptación del bucle es especialmente relevante para el propósito de este texto. El bucle se considera una forma narrativa muy vinculada a los videojuegos, puesto que da cuenta de un aspecto esencial de la interactividad, el aprendizaje de procedimientos según el entrenamiento repetitivo de habilidades para superar niveles y seguir avanzando hacia la meta, que tiene en el modelo PECMA flow una expresión privilegiada (Grodal, 2003). El tratamiento de la frecuencia, en términos narratológicos, ilustra bien los trasvases, dificultades e inevitables transformaciones a que se ve sometida un tipo de estructura narrativa cuando pasa de un medio interactivo -los videojuegos- a otro que no lo es -las películas-. Efectivamente, mientras que en innumerables videojuegos de aventura gráfica o acción, siempre en su modalidad de un solo jugador -nos referimos a juegos como Doom (1993-2016), Silent Hill (1999-2012), God of War (2005-2013), etc.- podemos hablar con propiedad de un relato repetitivo, en tanto que esta repetición se encuentra en el nivel del relato y no de la historia, puesto que la historia es una mientras que el relato, conducido por el jugador implicado según las posibilidades de las reglas del juego, es múltiple. Por decirlo de otra manera, el jugador repite el relato, pero no los personajes del juego, que viven, por decirlo así, cada repetición como la primera. En el cine, en cambio, en ausencia de un jugador implicado que impulsa activamente el relato, el espectador, mero sujeto pasivo, no tiene papel alguno en el desarrollo del bucle. Este papel lo llevan a cabo los personajes en el mundo de ficción de la película, de modo que la repetición está en el nivel de la historia -es intradiegética- y en el nivel del relato, en consecuencia, también. En términos narratológicos llamamos a estos casos singulativo anafórico, pero no relato repetitivo.

Particularmente interesante de este tipo de adaptación de la estructura narrativa es Scott Pilgrim vs. the World (2010). La película, sin ser adaptación directa de un videojuego, está, en su estructura y estética, claramente inspirada en los juegos de lucha tipo Tekken (1995-2009) o Street Fighter (1987-2016). Toma prestados tanto elementos de la interfaz -niveles de vida, sobreimpresiones de texto del tipo «you win» o «ko»- como estilos de lucha. Y remite también a la estructura de estos juegos, que en su modo historia imponen al jugador el objetivo de vencer a un determinado número de oponentes en sendas luchas cuerpo a cuerpo, del mismo modo que se le impone a Scott vencer a los siete «exnovios siniestros» de Ramona para poder seguir a su lado.

\subsection{Adaptaciones de la participación}

Participación y jugabilidad constituyen por derecho propio la característica más definitoria y distintiva de todo videojuego, como hemos visto, aquello que distingue a los videojuegos de las películas. Si bien las adaptaciones del mundo no ofrecían especiales dificultades, en tanto que los videojuegos narrativos ya influenciados por la narrativa cinematográfica contienen suficientes elementos adaptables, también hemos visto como las estructuras narrativas, aun pudiendo ser adaptadas, sufren transfiguraciones importantes, como en el relato repetitivo del bucle. Finalmente, la adaptación de la jugabilidad, de los aspectos más dinámicos de la narrativa del videojuego, puesto que supone la activación de las reglas, tendrá también en el cine una notable transformación. 
Las adaptaciones en términos de participación pueden dividirse a nuestro modo de ver en dos grupos. El primero, de tipo estético, toma prestados de los videojuegos estilos formales para trasladarlos a la mostración cinematográfica. El segundo toma elementos propios de la interfaz de los videojuegos para, integrados diegéticamente en el desarrollo mismo de la trama, constituirse en alegoría.

Los ejemplos más característicos del primer grupo pertenecen a ciertas apuestas de la forma fílmica por imitar, con menor o mayor fortuna, la estética de los videojuegos; en particular, el punto de vista en primera persona, un elemento muy característico de un determinado género, los First Person Shooter como Doom o Call of Duty (2003-2016) y que formalmente se ha constituido en seña de identidad del lenguaje de los videojuegos -una cámara virtual en primera persona, que recorre un espacio en plano secuencia-. Esta figura de estilo tiene relativamente fácil traslación al cine, en forma de cámara subjetiva y plano secuencia también. A.R. Galloway, en su análisis comparado del FPS y del subjetive shot cinematográfico, apunta que esa misma figura formal tiene, en la historia del cine y en la historia de los videojuegos, significados narrativos bien distintos, en tanto que

the subjective camera is largely marginalized in filmmaking and used primarily to effect a sense of alienated, disoriented, or predatory vision. Yet with the advent of video games, a new set of possibilities were opened up for the subjetive shot. In games the first-person perspective is not marginalized but instead is commonly used to achieve an intuitive sense of affective motion. It is but one of the many ways in which video games represent action. In other words, video games are the first mass media to effectively employ the first-person subjective perspective, whereas film uses it only for special ocasion [...]. Where film uses the subjetive shot to represent a problem with identification, games use the subjective shot to create identification. While film has thus far used the subjective shot as a corrective break through and destroy certain stabilizing elements in the film apparatus, games use the subjective shot to facilitate an active subject position that enables and facilitates the gamic apparatus (Galloway, 2006: 69).

Sin embargo, indudablemente por influencia de los videojuegos, el uso de la toma subjetiva parece haberse normalizado en cine, de modo que también en este medio no interactivo busca una mayor inmersión e identificación del espectador. Encontramos entonces la adaptación de elementos formales propios de los videojuegos al cine, como el caso de Doom, la película -su plano secuencia, puro gameplay, es una toma subjetiva que imita literalmente la perspectiva en primera persona tan característica de esta serie de videojuegos $y$, en definitiva, del género-, pero también como en el caso de The Revenant (2015), con una cámara en continuo movimiento y aunque no estrictamente en mostración subjetiva, con un uso de lentes gran angular que magnifican el espacio -y cuyas distorsiones de la imagen no sólo no están disimuladas sino que incluso parecen deliberadamente buscadas, algo poco habitual en el cine hasta ahora- de modo similar al que podemos encontrar en las últimas entregas de la serie de videojuegos Tomb Raider, como en Tomb Raider (2013) o en Rise of the Tomb Raider (2016).

Finalmente, podemos considerar un último tipo de adaptación, que utiliza elementos formales o de puesta en escena en forma alegórica, en un paralelismo entre las vivencias de los personajes en la película y determinados videojuegos, las acciones de cuyos personajes 
son conducidas por el jugador a través de la interfaz del juego, es decir, de la pantalla interactiva que permite manipular el mundo de juego desde el mundo de la vida. En tanto que la pantalla cinematográfica no es una interfaz, puesto que obviamente no permite la participación, la adaptación de la acción del jugador al cine tomará forma alegórica, es decir, una plasmación intradiegética de la acción del jugador, que en los videojuegos es siempre extradiegética. El recurso indirecto a la alegoría permite representar en cine aspectos de la jugabilidad de los videojuegos. Quizá el ejemplo más claro es Ben X (2007), una película sobre el jugador de un típico MMORPG -Massively Multiplayer Online Rol Playing Game- como pueda ser World of Warcraft (2004-), que supera sus dificultades en la vida a través de la inspiración y fuerza que encuentra en el juego. La película empieza en el videojuego Archlord (2005-2014), donde el protagonista, Ben, que sufre algún tipo de trastorno de personalidad que dificulta sus relaciones sociales, se siente realmente cómodo y feliz. Al abandonar el juego, y prepararse para ir a la escuela, se nos muestra a Ben «configurando» su vestuario y aspecto general, con elementos típicos de una interfaz como menús contextuales, cuadros de diálogo o listas desplegables que establecen, a un nivel intradiegético, una clara relación entre el mundo de juego y el mundo de vida de Ben.

También Chatroom (2010) puede incluirse en este grupo, con sus salas de chat visualizadas como abstractas habitaciones donde los personajes de la película, y sus avatares en el chat, se encuentran e interactúan.

Como se ve, nos referimos en este tipo de adaptación sobre todo al uso de aspectos formales que son transformados para su mostración cinematográfica, pero no a traslaciones al nivel del contenido, que ya hemos tratado en la adaptación del mundo. Una película como Los sustitutos (2009) no pertenecería a este grupo, puesto que si bien su argumento se inspira en la sustitución en la vida por parte de avatares, en cierto modo al estilo de The Sims (2000-2014) o Second Life (2003), se trata de un recurso sobre el mundo de la ficción y no sobre el modo en que la jugabilidad o participación en el mundo de un videojuego se adapta al mundo de una película.

\section{CONCLUSIONES}

Las relaciones del cine con otros medios son múltiples y diversas. El cine ha proporcionado a no pocos nuevos medios su lenguaje audiovisual y en parte también sus estructuras y sentido del espectáculo. No es raro que estos nuevos medios, como los videojuegos, deudores del cine en primera instancia, acaben por influirlo y transformar de muy distintas formas su lenguaje, estructuras y estilos. Entender el cine como un medio aislado, que se explica a sí mismo según su tradición, historia o géneros no da cuenta del complejo momento contemporáneo.

El análisis cinematográfico a la luz de las adaptaciones de los videojuegos al cine -en sus distintos aspectos de mundo de juego, reglas y estructura y participación y jugabilidad-, puede proporcionar una aportación a un mejor conocimiento del cine contemporáneo, como hemos visto, ineludiblemente influido por narrativas de muy distinto tipo, que toman del cine su lenguaje, estructura y estética pero lo transforman por virtud de las posibilidades de esos nuevos medios de comunicación. En ese retorno al cine, en forma de adaptaciones directas e indirectas, estamos más capacitados para entender las nuevas estrellas del cine -Lara Croft: Tomb Raider-, las nuevas estructuras -Scott Pilgrim vs. the World-y las nuevas 
alegorías -Ben $X$ - si rastreamos su relación con los contenidos del mundo, las estructuras y reglas y la jugabilidad y participación en los videojuegos, un medio ya no tan nuevo y que impone no pocos modos de consumo a espectadores/jugadores habituados a formas que deberían ser inevitablemente consideradas transgresiones desde el punto de vista de la narrativa clásica.

Parece en este sentido que la producción cinematográfica contemporánea, formando parte de la pantalla global o de la adaptación, supera modos de entender el cine anclados en el producto aislado y la narración transparente, unívoca y fiable, y cabe entenderla en complejas relaciones no solo intertextuales sino también irreversiblemente intermediales.

\section{BIBLIOGRAFÍA CITADA:}

AARSETH, E., «Storylines vs. gameworlds. Landscaping as narrative device?», 5enes Jornades sobre arte i multimèdia. Metanarrative(s)? Trobada internacional sobre el papel de l'art en el desenvolupament de narratives i sintaxis àudiovisuals i multimèdia. Fundació La Caixa, Barcelona, 2004.

ANYÓ, L., "Los videojuegos y la comunicación audiovisual», en REQUEIJO, P. \& GAONA, C. (Eds.), Contenidos innovadores en la Universidad actual, McGraw-Hill/Interamericana, Madrid, 2014, pp. 75-84.

ANYÓ, L., El jugador implicado. Videojuegos y narraciones, Laertes Ediciones, Barcelona, 2016.

COLOM, À. \& ANYÓ, L., "Argumentos para el clímax: Estrategias narrativas en el videojuego de acción y aventuras. ", en CUESTA, J. \& SIERRA, J. (Eds.), Videojuegos: arte y narrativa audiovisual, ESNE editorial, Madrid, 2015, pp. 69-85.

DE-FELIPE, F. \& GÓMEZ, I., Adaptación, Trípodos, Barcelona, 2008.

DELEYTO, C., Ángeles y demonios. Representaciones e ideología en el cine contemporáneo de Hollywood, Paidós, Barcelona, 2003.

GALLOWAY, A. R., Gaming. Essays on Algorithmic Culture, University of Minnesota Press, Minneapolis; London, 2006.

GRODAL, T., "Stories for eye, ear, and muscles: Video games, media, and embodied experiences», en WOLF, M. J. P. \& PERRON, B. (Eds.), The video game theory reader, Routledge, New York, 2003, pp. 126-156.

LIPOVETSKY, G. \& SERROY, J., La pantalla global. Cultura mediática y cine en la era hipermoderna, Anagrama, Barcelona, 2009.

MANOVICH, L., El lenguaje de los nuevos medios de comunicación. La imagen en la era digital, Paidós, Barcelona, 2005.

MARTÍNEZ-FABRE, M-P., «Trasladar la partida: Modelos y estrategias de la industria cinematográfica frente a la adaptación de un videojuego», en prensa, 2017.

METZ, C., El significante imaginario. Psicoanálisis y cine, Paidós, Barcelona, 2001.

MORIN, E., El cine o el hombre imaginario, Paidós, Barcelona, 2011.

MURRAY, J., Hamlet en la holocubierta. El futuro de la narrativa en el ciberespacio, Paidós, Barcelona, 1999.

PLANELLS, A. J., Videojuegos y mundos de ficción. De Super Mario a Portal, Cátedra, Madrid, 2015.

RIAMBAU, E., Hollywood en la era digital, Cátedra, Madrid, 2011. 
TASKER, Y., «Aproximación al nuevo Hollywood», en CURRAN, J., MORLEY, D. \& WALKERDINE,

V. (Eds.), Estudios culturales y comunicación. Análisis, producción y consumo cultural de las políticas de identidad y el posmodernismo, Paidós, Barcelona, 1998, pp. 323-346.

TRUFFAUT, F., El cine según Hitchcock, Alianza Editorial, Barcelona, 1984.

VILLALOBOS, J. M., Cine y videojuegos. Un diálogo transversal, Héroes de papel, Sevilla, 2014.

\section{FILMOGRAFÍA}

Al filo del mañana (D. Liman, 2014)

Alone in the dark (U. Boll, 2005)

Ben X (N. Balthazar, 2007)

Carretera perdida (D. Lynch, 1997)

Chatroom (H. Nakata, 2010)

club de la lucha, El (D. Fincher, 1999)

Donnie Darko (R. Kelly, 2001).

Dos vidas en un instante (P. Howitt, 1998)

Double Dragon (J. Yukich, 1993)

eXistenZ (D. Cronenberg, 1999)

Femme Fatale (B. de Palma, 2002)

Final Fantasy: la fuerza interior (H. Sakguchi, M. Sakakibara, 2001)

Funny Games (M. Haneke, 1997)

halcón maltés, El (J. Huston, 1941)

Hitman (X. Gens, 2007)

Identidad (J. Mangold, 2003)

King of Figther, The (G. Chan, 2010)

L.A. Confidencial (C. Hanson, 1997)

Lara Croft: Tomb Raider (S. West, 2001)

Max Payne (J. Moore, 2008)

Memento (Ch. Nolan, 2000)

Mortal Kombat (P.W.S. Anderson, 1995)

Mulholland Dr. (D. Lynch, 2002)

Need for Speed (S. Waugh, 2014)

Postal (U. Boll, 2007)

Prince of Persia: las arenas del tiempo (M. Newell, 2010)

reino de los cielos, El (R. Scott, 2005)

Resident Evil (P.W.S. Anderson, 2002)

Revenant, The (A. G. Iñárritu, 2015)

rules of attraction, The (R. Avary, 2002)

Run, Lola, Run (T. Tykwer, 1998)

Scott Pilgrim vs. the World (E. Wright, 2010)

Silent Hill (Ch. Gans, 2006)

Source Code (D. Jones, 2011)

Street Fighter: la última batalla (S. E. de Souza, 1994)

Sucker Punch (Z. Snyder, 2011)

Super Mario Bros (A. Jankel, R. Morton, 1993)

Quaderns, 12 (2017), pp. 11-25 
Swimming Pool (F. Ozon, 2003)

Tekken (D.H. Little, 2010)

\section{LUDOGRAFÍA}

Archlord (varios diseñadores, 2005-2014)

Assassin's Creed (Jade Raymond, 2007)

Doom, serie (varios diseñadores, 1993-2016)

God of War, serie (varios diseñadores, 2005-2013)

L.A. Norie (B. McNamara, 2011)

Rise of the Tomb Raider (B. Horton, D. Neuburger, M. Brinker, 2016)

Second Life (varios diseñadores, 2003- )

Silent Hill, serie (varios diseñadores, 1999-2012)

Sims, The, serie (varios diseñadores, 2000-2014)

Street Fighter, serie (varios diseñadores, 1987-2016)

Tekken, serie (varios diseñadores, 1995-2009)

The Last of Us (B. Straley, N. Druckmann, 2013)

Tomb Raider (N. Hughes, C. Barlog, D. Gallagher, 2013)

Tomb Raider, serie (varios diseñadores, 1996-2015)

Until Dawn (W. Byles, N. Bowen, 2015)

World of Warcraft (R. Pardo, J. Kaplan, T. Chilton, 2004) 